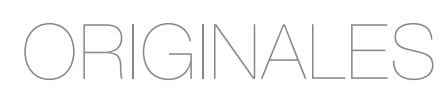

\title{
Alcances y límites de la asignación de presupuesto y financiamiento en posgrados del PNPC-CONACYT
}

FRANCISCA SILVA HERNÁNDEZ*

* Doctora en Métodos de Solución de Conflictos y Derechos Humanos. Universidad Juárez Autónoma de Tabasco, Tabasco, México. E-mail: francisca.silva@ujat.mx. ORCID: 0000-0003-3533-0002.GoogleScholar: https://scholar.google.es/citations?user=4QpMnMYAAAA\&hl=es. 


\section{COMO CITAR ESTE ARTÍCULO}

How to cite this article:

Silva, F. (2020). Alcances y límites de la asignación de presupuesto y financiamiento en posgrados del PNPC-CONACYT. Revista Perspectiva Empresarial, 7(2), 65-73.

Recibido: 24 de julio de 2020

Aceptado: 03 de octubre de 2020
RESUMEN El presente trabajo tiene como objetivo describir los alcances y límites que conlleva un posgrado integrado escolarizado orientado a la investigación a partir de las fuentes de financiamiento y asignación de presupuesto por recursos otorgados (recursos universitarios, subsidio estatal, subsidio federal) para los diversos gastos generados en la operatividad de los mismos. El método utilizado es de un estudio de caso de un posgrado escolarizado; de igual manera se aplicó el método documental que determinó las categorías de análisis descritas, que posteriormente se complementa en la sistematización y análisis de la información. Como resultado de la investigación se obtuvo que a partir del trabajo en colaboración entre el responsable académico y responsable administrativo de los posgrados se requiere una planeación estratégica a plazos que permita una eficacia en los indicadores mínimos requeridos por CONACYT en su proceso de evaluación. Finalmente la asignación y ejercicio de presupuesto al área de formación académica y de investigación para los posgrados es de vital importancia, permitiendo así su permanencia y ascenso en el PNPCCONACYT.

PALABRAS CLAVE posgrado, financiamiento, presupuesto, educación de calidad, sistema de calidad.

\section{Scope and limits of budget allocation and funding for PNPC- CONACYT postgraduate programs}

KEYWORDS Postgraduate, financing, budget, quality education, quality system.

ABSTRACT The aim of this paper is to describe the scope and limits of a research-oriented integrated school-based graduate program based on the financing sources and budget allocation for resources granted (university resources, state subsidy, federal subsidy) for the different expenses generated in its operation. The method used is a case study of a schoolbased postgraduate program; likewise, the documentary method was applied to determine the categories of analysis described, which is later complemented in the systematization and analysis of the information. As a result of the research, it was found that from the collaborative work between the academic and administrative people responsible for the postgraduate programs, a strategic planning with deadlines is required to allow efficiency in the minimum indicators required by CONACYT in its evaluation process. Finally, the allocation and exercise of budget to the area of academic and research training for postgraduate programs is of vital importance, thus allowing their permanence and promotion in the PNPC-CONACYT. 


\section{Alcance e limites da dotação e financiamento orçamentário nos programas de pós-graduação do PNPC-CONACYT}

RESUMO 0 objetivo deste trabalho é descrever a abrangência e os limites que uma pós-graduação integrada voltada para a pesquisa envolve a partir das fontes de financiamento e de destinação orçamentária dos recursos concedidos (recursos universitários, subsídio estadual, subsídio federal) para os diversos gastos gerados na operação do mesmo. 0 método utilizado é a partir de um estudo de caso de uma escola de pós-graduação; da mesma forma, foi aplicado o método documental que determinou as categorias de análise descritas, o qualé posteriormente complementado na sistematização e análise das informações. Como resultado da pesquisa, obteve-se que a partir do trabalho colaborativo entre o gestor acadêmico e o gestor administrativo dos cursos de pós-graduação, é necessário um planejamento estratégico em termos de tempo que permita uma efetividade nos indicadores mínimos exigidos pelo CONACYT em sua processo de avaliação. Por fim, a destinação e exercício do orçamento para a área de formação acadêmica e pesquisa para pós-graduados é de vital importância, permitindo assim sua permanência e promoção no PNPC-CONACYT.

PALAVRAS-CHAVE pós-graduação, financiamento, orçamento, educação de qualidade, sistema de qualidade. 


\section{Introducción}

De acuerdo con el Anuario Estadístico de Educación Superior de la Asociación Nacional de Universidades e Instituciones de Educación Superior -ANUIES-, durante el ciclo escolar 20182019, en México, hubo una matrícula existente de 267 estudiantes en elárea de posgrado (distribuidos en especialización, maestría y doctorado).

En México, el Consejo Nacional de Ciencia y Tecnología - CONACYT - desde hace cincuenta años tiene como objeto articular políticas públicas del gobierno federal y promover el desarrollo de la investigación científica, el desarrollo tecnológicoyla innovación con el fin de impulsar la modernización tecnológica del país. En este sentido el Programa Nacional de Posgrados de Calidad - PNPC - forma parte de la política pública de fomento a la calidad de los posgrados desde hace veintinueve años.

Al cierre de 2018 había en el padrón del PNPC un total de 2346 programas de posgrados de calidad acreditados, con un crecimiento del $7 \%$ en comparación con el primer semestre de ese mismo año. Respecto al impacto de los programas PNPC por área de conocimiento del Sistema Nacional de Investigadores - SNI-, el área de humanidades representaba el tercer lugar con un $34 \%$; ocupando los dos primeros lugares el área de medicina y de ciencias de la salud, así como el área de ingenierías (CONACYT, 2019).

Conforme al diagnóstico de los posgrados en México realizado por el Consejo Mexicano de Estudios de Posgrado - COMEPO — en la región sursureste durante el período 2014-2015 la asignación presupuestal debió ser de forma racional, equitativa y suficiente en todas las áreas que conforman el desarrollo del posgrado. Para 2019, de acuerdo a la declaratoria de COMEPO, se señala como una sitiuación compleja la asignación de recursos y recorte presupuestal; por tanto, afirma que:

la ley y en los presupuestos posteriores también se otorgue la debida prioridad al financiamiento de las instituciones públicas de educación superior, con especial énfasis en la formación de posgradoy en la investigación científica, tecnológicay orientada claramente a la innovación, incluyendo iniciativas que se impulsen en lugar de cancelarse. (p. 2)
Así, para el ingreso y permanencia de los posgrados en el PNPC hay que cumplir con criterios e indicadores mínimos establecidos en los anexos y marco de referencia; en el caso de un posgrado de investigación y con modalidad escolarizada, este debe alinearse a lo conducente en el Anexo "A" (CONACYT, 2019).

Un aspecto fundamental para cumplir con los criterios e indicadores de un posgrado perteneciente al PNPC es que la institución de educación superior - IES - apueste a la inversión educativa mediante diversos medios económicos que promuevan el avance y progreso del programa educativo hacia un alto impacto (Becerra, 2017). Lo anterior, puede proceder de aspectos como sistemas de aseguramiento y financiamiento a través de acciones concretas del programa educativo. Por ende, en este artículo, se exponen los alcances y límites de la asignación de recursos para desarrollar un programa educativo y cumplir con lo requerido por el CONACYT para la permanencia del posgrado en el PNPC.

\section{Materiales y métodos}

Este documento se basa en una metodología cualitativa (Garza, 2013), aplicando el método documental a partir de la consulta directa de fuentes de información primaria y secundaria (Villaseñor, 2008). A partir de la categorización de la información se procedió a la sistematización de la misma y al análisis concreto de la aplicabilidad de presupuestos en la inversión de la educación en el nivel superior.

El objeto de estudio fue un posgrado integrado escolarizado con orientación en la investigación en una IES pública de la región sur-sureste de México. Para comprender la dinámica del mismo se aplicó el método descriptivo y la consulta de todos aquellos planes y disposiciones reglamentarias y normativas de la IES; además se realizó el análisis de los recursos asignados y ejercidos en los programas educativos para poder alcanzar, mantener e innovar los posgrados acreditados en el CONACYT. 


\section{Resultados}

La Universidad Juárez Autónoma de Tabasco para 2019 contaba con 43 programas educativos de posgrado incorporados al PNPC-CONACYT. De estos, la División Académica de Ciencias Sociales y Humanidades - DACSyH - imparte 2 maestrías y 2 doctorados que se encuentran vigentes en el PNPC; estos, tienen la característica de posgrado integrado.

Al respecto, para la calidad de estos posgrados existen dos funciones que es importante distinguir y que el programa educativo debe cumplir de acuerdo al CONACYT: (i) la responsabilidad académica del posgrado, el que lo asume como responsable tiene la función de crear y dar cabal seguimiento a toda actividad académica que fortalezca las líneas de generación y aplicación del conocimiento (investigación, producción, vinculación, movilidad, estancias, comités tutoriales, fortalecimiento del desempeño del becario con actividades extracurriculares) de estudiantes y profesores; (ii) la responsabilidad administrativa del desarrollo del posgrado, esa función recae en la Coordinación de Posgrado de la División Académica - que depende del área denominada "Programa Nacional de Posgrados de Calidad"- - Las funciones que debe desempeñar son aquellas dirigidas a atender y gestionar los trámites de proceso de admisión ante las instancias administrativas correspondientes (relación de interlocución); generar formatos, guías o procesos que garanticen la permanencia del estudiante; facilitar trámites que dispongan de tiempo límite por parte de la Dirección de Posgrado o el CONACYT; prever la comunicación continua y permanente con las áreas de finanzas, planeación y evaluación institucional, servicios escolares, secretaría de investigación, posgrado y vinculación, dirección de tecnologías de la información e innovación.

El papel de esas figuras se conjuga de forma dinámica y colaborativa, situación que ha llevado a los posgrados a permanecer y ascender de nivel en el PNPC-CONACYT. Es importante señalar que la responsabilidad administrativa conlleva también una corresponsabilidad por parte de la IES, derivada de la carta de compromiso que como institución educativa presenta ante el CONACYT.

Es importante considerar que desde 2014 hasta la fecha los posgrados de la DACSyH han sido apoyados a través de recursos federales, provenientes en su momento del Programa de Fortalecimiento de la Calidad en Instituciones Educativas -PROFOCIE - y el Programa de Fortalecimiento de la Calidad Educativa -PFCE-. Ambos programas coadyuvaron al proceso de desarrollo de los posgrados. En la siguiente tabla se señalan todas las actividades académicas y de investigación realizadas con el financiamiento de estos recursos:

Tabla 1. Actividades académicas y de investigación en posgrado PNPC período 2014-2018

\begin{tabular}{ll}
\hline Año & \multicolumn{1}{c}{ Apoyo institucional y del CONACYT } \\
\hline 2014 & $\begin{array}{l}\text { Adecuación de instalaciones de edificio para los posgrados de nueva creación pertenecientes al PNPC } \\
\text { Participación de siete profesores investigadores de universidades nacionales e internacionales } \\
\text { De } 39 \text { estudiantes matriculados en los posgrados en este año, } 32 \text { fueron beneficiados con una beca CONACYT }\end{array}$ \\
\hline 2015 & $\begin{array}{l}\text { Beca PISA a estudiantes de los posgrados } \\
\text { Apoyo y facilidades en actividades académicas durante el ciclo escolar agosto 2014-enero 2015 (tres talleres, tres } \\
\text { Conferencias y un foro) } \\
\text { Apoyo para realización de estancias académicas profesores-estudiantes }\end{array}$ \\
\hline $2016 \quad \begin{array}{l}\text { Desarrollo de 30 actividades académicas } \\
\text { Apoyo del CONACYT e institucional de manera económica para realizar estancias de investigación } \\
\text { Egreso de estudiantes de maestría } \\
\text { Cinco estudiantes fueron beneficiados con la beca institucional PISA }\end{array}$ \\
\hline
\end{tabular}




\begin{tabular}{ll}
\hline Año & \multicolumn{1}{c}{ Apoyo institucional y del CONACYT } \\
\hline 2017 & $\begin{array}{l}\text { Se fortalece la IES a través de la continuidad de formación académica y de investigación de los docentes en los } \\
\text { posgrados del PNPC; en 2017, egresaron y obtuvieron el grado de ‘Doctor’ diez profesores de la División Académica }\end{array}$ \\
& $\begin{array}{l}\text { Apoyo institucional para validar la participación y renovar la vigencia de los programas educativos de maestría } \\
\text { 33 actividades académicas (foros y congresos) }\end{array}$ \\
\hline $2018 \quad \begin{array}{l}\text { Actividades académicas (foros y congresos) } \\
\text { Once estudiantes con acción de movilidad académica } \\
\text { Publicaciones } \\
\text { Proceso de admisión }\end{array}$ \\
\end{tabular}

Fuente: elaboración propia por parte de la autora.

Para el desarrollo de estas acciones académicas y de investigación, el responsable académico y el responsable administrativo coordinan sus funciones; procediendo a realizar una planeación a corto, mediano y largo plazo que permita cumplir con el "Plan de Mejora" que se entrega al CONACYT.
Para ello es importante señalar que durante el período 2014-2018 se ejerció el siguiente recurso para la operatividad de los programas educativos:

Tabla 2. Asignación y ejercicio de presupuesto en posgrado PNPC período 2014-2018

\begin{tabular}{|c|c|c|}
\hline Año & Concepto & Monto \\
\hline \multirow[t]{4}{*}{2014} & Programa para la operatividad para los programas pertenecientes al PNPC & \multirow{4}{*}{$\$ 508.896$} \\
\hline & Pago de honorarios asimilables al salario de maestrías y doctorados & \\
\hline & Instalación de conectividad del área del PNPC & \\
\hline & Operatividad para los programas & \\
\hline \multirow[t]{3}{*}{2015} & Operatividad para los programas & \multirow{3}{*}{$\$ 732.517,88$} \\
\hline & Pago de honorarios asimilables al salario de maestrías y doctorados & \\
\hline & Asegurar la calidad de los programas PROFOCIE & \\
\hline \multirow[t]{2}{*}{2016} & Asegurar la calidad de los programas & \multirow{2}{*}{$\$ 369.087,88$} \\
\hline & Operatividad para los programas & \\
\hline \multirow[t]{2}{*}{2017} & Asegurar la calidad de los programas & \multirow{2}{*}{$\$ 1 ' 024.522$} \\
\hline & Pago de honorarios de servicios profesionales a profesores de posgrado & \\
\hline \multirow[t]{3}{*}{2018} & Apoyo a estudiantes de posgrado para la realización de eventos académicos & \multirow{3}{*}{$\$ 470.588$} \\
\hline & Apoyo a estudiantes que participarán en eventos académicos & \\
\hline & Acervo bibliográfico & \\
\hline
\end{tabular}

Fuente: elaboración propia por parte de la autora.

Es importante puntualizar que a través de las asignaciones de beca nacional y de movilidad nacional e internacional que otorga el CONACYT, los estudiantes han podido financiar su educación del programa educativo. La IES por medio de sus posgrados participa en las convocatorias que emite el CONACYT, así como aquellas que puedan beneficiar el desarrollo y fortalecimiento de los posgrados. Por mencionar, durante estos seis años se ha podido colaborar con investigadores que participaron en estancias posdoctorales y tuvieron participación significativa en los indicadores de los posgrados. 
Otros mecanismos de fuente de financiamiento para los estudiantes y profesores de los posgrados han sido:

(i) Beca del Programa Institucional de Superación Académica - PISA - para estudiantes: esta otorgaba un estímulo económico mensual a estudiantes egresados de la Universidad Juárez Autónoma de Tabasco que estuvieran cursando un posgrado con la condicionante de mantener sin interrupción su trayectoria académica; egresar en tiempo de acuerdo al plan de estudios del posgrado sin haber reprobado asignaturas, así como cumplir con la eficiencia terminal acorde a la convocatoria en la que hayan participado. Esta beca se suspendió en 2017 por situaciones de austeridad universitaria.

(ii) Beca del Programa Institucional de Superación Académica - PISA - para profesores: esta beca es dirigida a todo profesor de la universidad que desea continuar con sus estudios de posgrado. Tiene la finalidad de apoyar con la descarga académica del profesor para realizar su formación continua.

(iii) Programa de intercambio académico institucional: este programa permite promover a través de los convenios de colaboración, estancias a estudiantes y profesores en otras instituciones educativas nacionales o internacionales.

(iv) CONACYT (beca nacional): se postula a estudiantes inscritos en el posgrado a participar por la beca nacional que otorga el CONACYT, cuyo apoyo económico es mensual, quedando condicionados al "Reglamento de Becas" del CONACYT y a toda la normativa que determine las condicionantes de la beca. En este rubro, estudiantes indígenas y madres solteras han participado en convocatorias a fin a su condición. De igual manera con el objeto de cumplir con el indicador de 'movilidad' se postula a estudiantes a las becas de estancias nacionales e internacionales; hoy en día, denominadas becas de movilidad.

(v) Becas de asociaciones, programas y fundaciones no gubernamentales: este tipo de financiamiento es conforme a convocatorias dirigidas a temas de líneas de investigación específicas, a temas relevantes conforme a situaciones complejas de sectores vulnerables. (vi) Sector gubernamental y privado: este tipo de financiamiento va dirigido a personas o grupos de personas con la necesidad de formación y actualización continua por parte de la institución para su personal.

(vii) Recursos propios: en este criterio el estudiante absorbe el costo total del posgrado (proceso de admisión, ingreso, permanencia, egreso y titulación). Aun cuando se ha beneficiado en su mayoría a estudiantes con beca CONACYT, hay estudiantes que por decisión propia deciden cursar el posgrado sin requerir esta beca.

\section{Discusión}

Acorde al presupuesto de egresos de la federación para el ejercicio fiscal 2020 en México, el CONACYT tuvo previsto en los gastos programables de becas de posgrado y apoyos a la calidad un monto total de \$25.658.798.449.

Aplicando principios de equidad e igualdad en cuanto a erogaciones para la igualdad entre mujeres y hombres, el CONACYT ejerció en el 2020 un monto total de $\$ 4.880 .050 .983$; los cuales están distribuidos en dos programas, el primero "Apoyos para actividades científicas, tecnológicas y de innovación" y el segundo "Becas de posgrado y apoyos a la calidad"; este último con un monto total de \$ 4.766.467.275. Asimismo, en el anexo de recursos para la adaptación y mitigación de los efectos del cambio climático, el CONACYT también previó "becas de posgrado y apoyos de calidad" por un monto total de $\$ 113.861 .093$. Por consecuente, el "Programa de Becas de Posgrado y Apoyos a la Calidad" contó para 2020 con un presupuesto de \$10.097,1 millones. La meta prevista era apoyar 59190 becas.

Lo que significa que en los dos períodos de solicitud debecasnacionales en 2020, los estudiantes participaron sin situación alguna que hiciera compleja su asignación de beca. Además representa una fuente de inversión a su educación y financiamiento de la misma. Por parte de la IES se apoya a los posgrados con la asignación de presupuesto federal en el "Programa de Fortalecimiento a la Excelencia Educativa 2020-2021". Igualmente se contempla 
que por la recaudación de recursos propios como inscripciones, reinscripciones, el programa educativo contara con los suministros necesarios para llevar a cabo de forma eficiente el desarrollo de los mismos.

En México, el tema de la austeridad está representando nuevos procesos de adaptación y cambios complejos (Mendoza, 2019). En estos posgrados integrados ha variado el presupuesto asignado conforme a los planes y políticas públicas en el país y en la región. Afortunadamente los posgrados han sabido aprovechar, explotar y potencializar los recursos asignados con base a la planeación de las actividades académicas y de investigación que se contemplan en el plan de trabajo sustantivo en plazos definidos. El ejercicio del presupuesto lleva una planeación que impacte de manera colectiva diversos indicadores del PNPC-CONACYT para su permanencia y ascenso de nivel. Por citar solo un ejemplo, la acción de movilidad permite afianzar lazos de colaboración mediante convenios generales y/o específicos para el intercambio académico, estancias, publicaciones colectivas y vinculación; todo esto derivado de la acción de 'movilidad', la cual conlleva el ejercicio de un presupuesto.

\section{Lo anterior, permite que se}

\begin{abstract}
garantice la continuidad de los programas de apoyo al posgrado en CONACYT, que han resultado claramente exitosos para la formación de los recursos humanos de alto nivel en el país, realizando los ajustes y re-orientación procedentes, a partir de análisis cuidadosos, como es el caso del PNPC, los programas de becas nacionales y al extranjero, las becas mixtas y apoyo a la movilidad, el S.N.I.; y se brinde además apoyo directo a los planes de mejora de los programas altamente pertinentes y al surgimiento de otros de igual relevancia, ya sea por parte del CONACYT o desde la SEP directamente. (COMEPO, 2019, p. 2)
\end{abstract}

Este señalamiento que hace el COMEPO distingue la necesidad imperante por parte de las universidades de potencializar los posgrados pertenecientes al PNPC, invirtiendo igual o mayor presupuesto en el desarrollo de los mismos; así, la Universidad Juárez Autónoma de Tabasco previó está situación para los 43 programas educativos de posgrado que tiene incorporados en el PNPCCONACYT.

\section{Conclusiones}

La función administrativa de los posgrados conlleva una planeación, organización, disciplina y un plan de trabajo a corto, mediano y largo plazo que permita la permanencia y el ascenso de nivel de los programas educativos. El trabajo colaborativo realizado por el responsable académico y el responsable administrativo permite una integración sustancial de las fortalezas y debilidades del posgrado, esto los lleva a trabajar en líneas de acción concretas para acrecentar y fortalecer los indicadores.

Se reconoce a la Universidad Juárez Autónoma de Tabasco el apoyo sustancial que como IES ha proporcionado a los posgrados pertenecientes al PNPC-CONACYT, pues en el caso de la DACSyH creó la infraestructura y el equipamiento necesarios para el desarrollo de los mismos; de igual manera ha fortalecido el núcleo académico básico con producción científica de alto impacto en obras individuales o colegiadas. La vinculación aliada con la movilidad ha causado un impacto importante que permea en los demás indicadores del programa educativo. Por ello la inversión de calidad educativa en posgrados PNPC debe considerar asignación y ejercicio del presupuesto en esos dos importantes indicadores; los cuales en conjunto con una planeación a corto, mediano y largo plazo mejorarán la calidad en la formación continua de estudiantes y profesores del posgrado.

\section{Referencias}

ANUIES. (2019). Anuario estadístico. Ciclo escolar 2018-2019. Ciudad de México, México: ANUIES.

Becerra, A. (2017). Alcances y limitaciones del posgrado en la Universidad Autónoma de Nayarit. Revista de la Educación Superior, 46(183), 105-121.

COMEPO. (2019). Declaratoria de Villahermosa. Villahermosa, México: COMEPO.

CONACYT. (2019). Programa Nacional de Posgrados de Calidad. Recuperado de http://svrtmp. 
main.conacyt.mx/ConsultasPNPC/images / infografia\%20PNPC.jpg.

CONACYT. (2019). Anexo "A" programas de orientación a la investigación. Versión 6.1. Recuperado de https://www.conacyt.gob. $\mathrm{mx} /$ index.php/sni/convocatorias-conacyt/ convocatorias-pnpc/anexos-pnpc/19427anexo-a-orientacion-profesional/file.

Garza, A. (2013). Manual de técnicas de investigación para estudiantes de ciencias sociales $y$ humanidades. Ciudad de México, México: COLMEX.

Mendoza, R.J. (2019). Presupuesto federal de educación superior en el primer año del gobierno de Andrés Manuel López Obrador: negociaciones y retos. Revista de la Educación Superior, 49(191), 51-82.

Villaseñor, R.I. (2008). Metodología para guías de fuentes de información. Investigación Bibliotecológica, 22(46), 113-138. 